\title{
VIBRATION SUPPRESSION SHAPE FILTER GENERATION FROM WINDOW FUNCTIONS
}

\author{
Li Zhou, Eduardo A. Misawa \\ School of Mechanical and Aerospace Engineering, Oklahoma \\ State University, Stillwater, OK 74078-5016, USA
}

\begin{abstract}
A control profile is generated which suppresses one or more resonant dynamics in a flexible dynamic system. This control profile can be used as a velocity profile, or as a shape filter to an arbitrary control command. The robustness can be arbitrarily improved, which brings about a smoother profile. The technique can be applied to both open-loop and closed-loop systems. Copyright (C)2005 IFAC
\end{abstract}

Keywords: Flexible dynamic system, residual vibration control

\section{INTRODUCTION}

Control of flexible structures has been extensively studied in recent years. Flexible structures such as high-speed disk drive actuators require extremely precise positioning under very tight time constraints. Whenever a fast motion is commanded, residual vibration in the flexible structure is induced, which increases the settling time. One solution is to design a closed-loop controller to damp out vibrations caused by the command inputs and disturbances to the plant. However, the resulting closed-loop response may still be too slow to provide an acceptable settling time, and the closed-loop control is not able to compensate for high frequency residual vibration which occurs beyond the closed-loop bandwidth. An alternative approach is to develop an appropriate reference trajectory that is able to minimize the excitation energy imparted to the system at its natural frequencies.

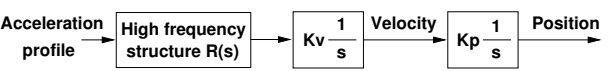

Fig. 1. A typical mechanical flexible system.

Fig. 1 shows a typical mechanical flexible system, where $\frac{1}{s}$ is an integrator, $K_{v}$ is a velocity constant

\footnotetext{
1 Supported by the National Science Foundation, grant number 9978748, and Seagate Technology LLC of Oklahoma City, Oklahoma.
}

gain, and $K_{p}$ is a position constant gain. The high frequency modes can be described as a transfer function $R(s)=\lim _{n \rightarrow \infty} \frac{b_{n} s^{n}+b_{n-1} s^{n-1}+\cdots+b_{1} s+1}{a_{n} s^{n}+a_{n-1} s^{n-1}+\cdots+a_{1} s+1}$ in which an infinite number of lightly damped resonant structures is possible. The goal of vibration suppression trajectory generation is to find a fast input trajectory, under some physical constraint, with minimum possible residual vibration. The position reference input can be generated from a step movement command $s(t)=S$. $1(t)$, through a finite support filter, $f(t), 0 \leq t \leq T$, where $T$ is the time duration of the finite support filter. To guarantee that the filtered command reaches the same set point as the step movement command, the integral of $f(t)$ must be equal to 1 , i.e., $\int_{0}^{T} f(t) d t=1$. This finite support filter $f(t), 0 \leq t \leq T$, which generates a vibration suppression position reference profile is called a "vibration suppression shape filter", or simply a "shape filter."

To suppress all the high frequency resonant dynamics in a flexible system, Zhou and Misawa (Zhou and Misawa, 2005b) have proposed vibration suppression shape filter and control profile generation based on optimal energy concentration functions. In practical system, a lower resonance frequency mode may exist which is located far from the high frequency resonance modes. If the low frequency is chosen to be a bandwidth in (Zhou and Misawa, 2005b) for the vibra- 
tion suppression control profile generation, the time duration of the profile is inefficiently increased. In this case, Zhou and Misawa (Zhou and Misawa, 2005a) have proposed vibration suppression shape filter and control profile generation for a specific resonance mode. In (Zhou and Misawa, 2005a), a vibration suppression shape filter and a vibration suppression control profile were generated based on a rectangle window and the continuous-time Input Shaping ${ }^{\circledR}$ (Singer and Seering, 1990) was shown to be a special case of the discrete-time rectangle window based shape filter with a particular sampling period. In this paper, vibration suppression shape filter and control profile generation based on other window functions is studied.

\section{VIBRATION SUPPRESSION SHAPE FILTER FOR A SPECIFIC RESONANCE MODE}

From the analysis of the relationship between control input and residual vibration of a damped resonant mode with the natural frequency $\omega_{i}$ and damping ratio $\zeta_{i}$, the following conclusion is drawn (Zhou and Misawa, 2005a).

Conclusion 2.1. If there exists a finite support base function $h(t), 0 \leq t \leq T_{0}$, such that $H\left(\omega_{d i}\right)=$ $H\left(\sqrt{1-\zeta_{i}^{2}} \omega_{i}\right)=0$, then, $h(t)$ may have two possible properties:

(1) The function $\frac{h(t)}{e^{\zeta_{i} \omega_{i} t}}$ is a control profile candidate to eliminate the residual vibration caused by the resonant mode with the natural frequency $\omega_{i}$ and the damping ratio $\zeta_{i}$.

(2) The function $\frac{h(t)}{e^{\zeta_{i} \omega_{i} t}}$ with a constraint $\int_{0}^{T_{0}} \frac{h(t)}{e^{\zeta_{i} \omega_{i} t}} d t$ $=1$, is a vibration suppression shape filter that can be used to filter out an arbitrary control profile, and the shaped control profile eliminates the residual vibration caused by the resonant mode with the natural frequency $\omega_{i}$ and the damping ratio $\zeta_{i}$.

The proof of Conclusion 2.1 is given in (Zhou, 2005). The philosophy of Conclusion 2.1 advocates that a shape filter should be able to be both a traditional shaping filter, as well as a possible command signal in its own right. Let $f_{1}(t)=\frac{h(t)}{e^{c_{i} \omega_{i} t}}$, then robustness of the properties in Conclusion 2.1 can be improved by the following filter operation,

$$
f_{n}(t)=\int_{0}^{t} f_{n-1}(t-\tau) f_{1}(\tau) d \tau, \quad n \geq 2,
$$

and the resultant spectrum of $f_{n}(t)$ is $F_{n}(\omega)=$ $F_{1}^{n}(\omega)$. Here, the control profile or shape filter $f_{1}(t)$ in (1) is said to have the robustness of order 1 . The control profile or shape filter $f_{n}(t)$ generated from the filter operation in (1) is said to have the robustness of order $n$. In the case that $f(t)$ is a non-continuous impulse function, the robustness improvement method (1) becomes the idea advocated by Singer and Seering (Singer and Seering, 1990).

In (Zhou and Misawa, 2005a), a vibration suppression shape filter and a vibration suppression control profile were generated based on a rectangle window. In the following sections, smooth shape filter generation based on other window functions is studied. Instead of analyzing the continuous-time case, the discrete-time case is directly generated.

\section{SHAPE FILTER GENERATION USING HANNING WINDOW}

If the sampling period is $T_{s}$ sec and the total discretetime sequence has $M+1$ impulses, the Hanning window function is

$$
h[k]= \begin{cases}0.5-0.5 \cos (2 \pi k / M), & \text { if } 0 \leq k \leq M \\ 0, & \text { otherwise }\end{cases}
$$

If $H\left(\omega_{d i} T_{s}\right)=H\left(\sqrt{1-\zeta_{i}^{2}} \omega_{i} T_{s}\right)=0$, then $\omega_{d i} T_{s}=$ $\frac{4 \pi}{M}$ and $M=\frac{4 \pi}{\omega_{d i} T_{s}}$. If $M$ is a positive integer, a smooth shape filter can be generated as $f[k]=$ $\frac{h[k] / e^{\zeta_{i} \omega_{i} k T_{s}}}{\sum_{m=0}^{M} h[m] / e^{\zeta_{i} \omega_{i} m T_{s}}}$. To improve robustness, the filter operation in (1) is performed. Let $f_{1}[k]=f[k]$, then robust shape filters $f_{2}[k], f_{3}[k], \ldots$ can be generated following the filter operation in (1). Since the sequence $h[k]$ is known, the sequence $f[k]$ can be generated through a simple numerical calculation.

For a second-order harmonic oscillator of the natural frequency $\omega_{i} \mathrm{rad} / \mathrm{sec}$ and the damping ratio $\zeta_{i}$, i.e., $\frac{\omega_{i}^{2}}{s^{2}+2 \zeta_{i} \omega_{i} s+\omega_{i}^{2}}$, the magnitude of the total response immediately after the $N^{t h}$ impulse is given by (Singer and Seering, 1990)

$$
\begin{aligned}
V_{a m p}\left(\omega_{i}, \zeta_{i}\right) & =e^{-\zeta_{i} \omega_{i} t_{N}} \frac{\omega_{i}}{\sqrt{1-\zeta_{i}^{2}}} \\
& \sqrt{\left(A C\left(\omega_{i}, \zeta_{i}\right)\right)^{2}+\left(A S\left(\omega_{i}, \zeta_{i}\right)\right)^{2}},
\end{aligned}
$$

where

$$
\begin{aligned}
A C\left(\omega_{i}, \zeta_{i}\right) & =\sum_{k=1}^{N} A_{k} e^{\zeta_{i} \omega_{i} t_{k}} \cos \left(\omega_{i} \sqrt{1-\zeta_{i}^{2}} t_{k}\right), \\
A S\left(\omega_{i}, \zeta_{i}\right) & =\sum_{k=1}^{N} A_{k} e^{\zeta_{i} \omega_{i} t_{k}} \sin \left(\omega_{i} \sqrt{1-\zeta_{i}^{2}} t_{k}\right) .
\end{aligned}
$$

The variables $A_{k}$ and $t_{k}$ are the amplitude and time location at which the impulse occurs, $N$ is the total number of impulses, and $t_{N}$ is the time of the last impulse. The sensitivity of the impulse shape filter can be displayed graphically by a sensitivity curve: a plot of residual vibration amplitude versus frequency error. Let $q=\omega_{\text {actual }} / \omega_{\text {model }}$, (3) becomes

$$
\begin{array}{r}
V_{a m p}\left(q \omega_{i}, \zeta_{i}\right)=e^{-\zeta_{i} q \omega_{i} t_{N}} \frac{q \omega_{i}}{\sqrt{1-\zeta^{2}}} \\
\sqrt{\left(A C\left(q \omega_{i}, \zeta_{i}\right)\right)^{2}+\left(A S\left(q \omega_{i}, \zeta_{i}\right)\right)^{2}},
\end{array}
$$


where $\omega_{i}=\omega_{\text {model }}, \omega_{\text {actual }}=q \omega_{i}, A C\left(q \omega_{i}, \zeta_{i}\right)=$ $\sum_{k=1}^{N} A_{k} e^{\zeta_{i} q \omega_{i} t_{k}} \cos \left(q \omega_{i} \sqrt{1-\zeta_{i}^{2}} t_{k}\right)$, and $A S\left(q \omega_{i}\right.$, $\left.\zeta_{i}\right)=\sum_{k=1}^{N} A_{k} e^{\zeta_{i} q \omega_{i} t_{k}} \sin \left(q \omega_{i} \sqrt{1-\zeta_{i}^{2}} t_{k}\right)$. Since for any finite impulse shape filter $f(t), 0 \leq t \leq$ $T_{0}$, the integral of $f(t)$ is $\int_{0}^{T_{0}} f(t) d t=1$ and the rigid body movement amplitude can be assumed as $\int_{0}^{T_{0}} f(t) d t=1$. The residual vibration level can be defined as a percentage of the rigid body motion amplitude, i.e., $\frac{V_{a m p}\left(q \omega_{i}, \zeta_{i}\right)}{\int_{0}^{T_{0}} f(t) d t}=V_{a m p}\left(q \omega_{i}, \zeta_{i}\right)$. For the impulse shape filter case, $\int_{0}^{T_{0}} f(t) d t=\sum_{k=1}^{N} A_{k}=$ 1. The residual vibration level (4) can be plotted for the Hanning based shape filter $f_{1}[k]$ and rectangle based shape filter $f_{1}[k]$. The sampling period $T_{s}$ is chosen to be $\frac{\pi}{100 \omega_{d i}}$. Fig. 2 shows the sensitivity curve of the rectangle based shape filter (Zhou and Misawa, 2005a) with $\omega_{\text {model }}=1 \mathrm{rad} / \mathrm{sec}$ and different damping $\zeta_{i}=0,0.05,0.2$. Fig. 3 shows the sensitivity curve of the Hanning based shape filter $f_{1}[k]$ with $\omega_{\text {model }}=1 \mathrm{rad} / \mathrm{sec}$ and different damping $\zeta_{i}=$ $0,0.05,0.2$. Clearly, the robustness of the Hanning based shape filter is increased both at the model natural frequency and the unmodeled high frequency.

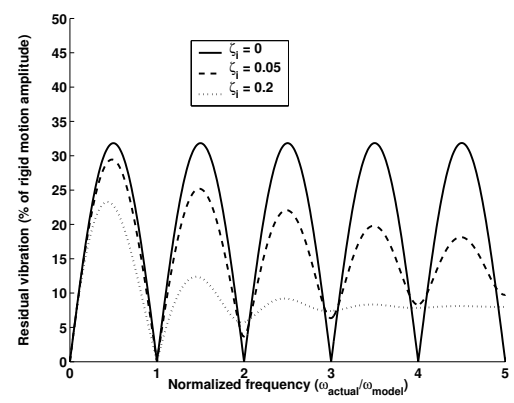

Fig. 2. Rectangle based shape filter $f_{1}[k]$ sensitivity plot versus actual natural frequency.

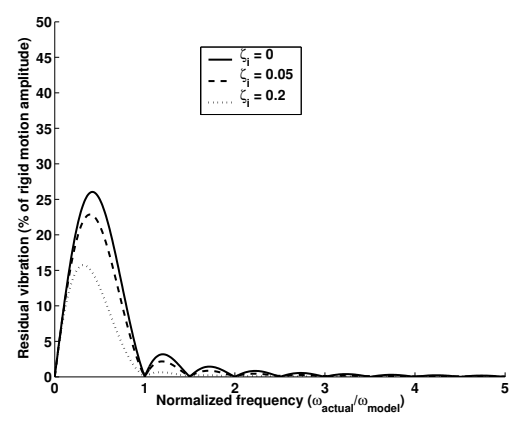

Fig. 3. Hanning based shape filter $f_{1}[k]$ sensitivity plot versus actual natural frequency.

The definition in (4) is different from the sensitivity concept of Singer (Singer and Seering, 1990) and Singhose (Singhose, 1997). In their definition, the sensitivity curve is expressed as the magnitude of the total response immediately after the $N^{t h}$ impulse divided by the magnitude of the response with unit impulse occurring at time 0 . Since the magnitude of the response with unit impulse occurring at time 0 is given by $\frac{\omega_{i}}{\sqrt{1-\zeta_{i}^{2}}}$, their definition of residual vibration level is $\frac{V_{a m p}\left(q \omega_{i}, \zeta_{i}\right)}{\frac{\omega_{i}}{\sqrt{1-\zeta_{i}^{2}}} \sum_{k=1}^{N} A_{k}}$ or simply

$$
e^{-\zeta_{i} q \omega_{i} t_{N}} \sqrt{\left(A C\left(q \omega_{i}, \zeta_{i}\right)\right)^{2}+\left(A S\left(q \omega_{i}, \zeta_{i}\right)\right)^{2}}
$$

because $V_{a m p}\left(q \omega_{i}, \zeta_{i}\right)$ exactly has the term $\frac{\omega_{i}}{\sqrt{1-\zeta_{i}^{2}}}$ and $\sum_{k=1}^{N} A_{k}=1$. It is known that the magnitude of the response with unit impulse occurring at time 0 , which is $\frac{\omega_{i}}{\sqrt{1-\zeta_{i}^{2}}}$, linearly increases with respect to actual undamped natural frequency $\omega_{i}$ if $\zeta_{i}$ is assumed to be a constant. Therefore, the definition (5) does not express the absolute residual vibration magnitude. For example, the definition (5) shows that the residual vibration level is $100 \%$ when the actual undamped natural frequency is 0 . However, the true magnitude of the residual vibration given in (4) is 0 when the actual undamped natural frequency is 0 .

Since the Hanning function starts and ends at zero, the Hanning based shape filter $f_{1}[k]$ can be used as a velocity candidate. Fig. 4 shows the rigid body acceleration, velocity, and position profiles generated from the Hanning based shape filter $f_{1}[k]$, with one resonant mode with parameters $\omega_{i}=1 \mathrm{rad} / \mathrm{sec}$ and damping ratio $\zeta_{i}=0.2$. Discrete-time shape filter generation for an arbitrary sampling period can be implemented following the procedure in (Zhou, 2005).

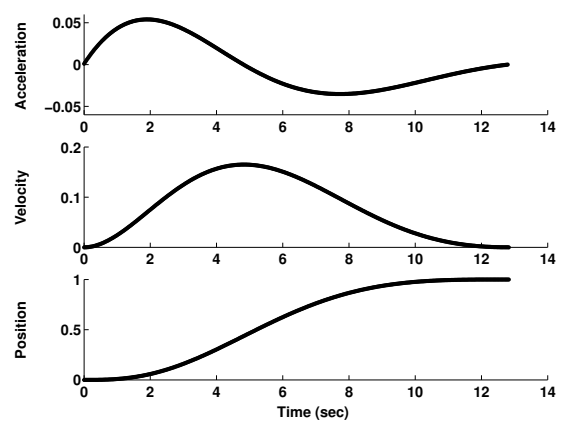

Fig. 4. Acceleration, velocity and position profiles from the Hanning based shape filter $f_{1}[k]$ with $\omega_{i}=1 \mathrm{rad} / \mathrm{sec}$ and $\zeta_{i}=0.2$.

\section{SHAPE FILTER GENERATION USING BLACKMAN WINDOW}

If the sampling period is $T_{s}$ sec and the total discretetime sequence has $M+1$ impulses, the Blackman window function is

$h[k]=\left\{\begin{array}{cl}0.42-0.5 \cos (2 \pi n / M) & \\ +0.08 \cos (4 \pi k / M), & \text { if } 0 \leq k \leq M, \\ 0, & \text { otherwise. }\end{array}\right.$

If $H\left(\omega_{d i} T_{s}\right)=H\left(\sqrt{1-\zeta_{i}^{2}} \omega_{i} T_{s}\right)=0$, then $\omega_{d i} T_{s}$ $=\frac{6 \pi}{M}$ and $M=\frac{6 \pi}{\omega_{d i} T_{s}}$. If $M$ is a positive integer, a smooth shape filter can be generated as $f[k]=$ 
$\frac{h[k] / e^{\zeta_{i} \omega_{i} k T_{s}}}{\sum_{m=0}^{M} h[m] / e^{\zeta_{i} \omega_{i} m T_{s}}}$. Again, it is not necessary to derive the explicit form for $f[k]$. Since sequence $h[k]$ is known, the sequence $f[k]$ can be generated through a simple numerical calculation. Let $f_{1}[k]=f[k]$, then robust shape filters $f_{2}[k], f_{3}[k], \ldots$ can be generated following the filter operation in (1).

\section{CAN HAMMING WINDOW BE USED TO SHAPE FILTER GENERATION?}

If the sampling period is $T_{s}$ sec and the total discretetime sequence has $M+1$ impulses, the Hamming window function is

$$
h[k]= \begin{cases}\frac{25}{46}-\frac{21}{46} \cos (2 \pi k / M), & \text { if } 0 \leq k \leq M \\ 0, & \text { otherwise }\end{cases}
$$

By calculation (Zhou, 2005), equation $H(\omega)=0$ has no solution and the shape filter cannot be generated from Hamming window in theory by following Conclusion 2.1. However, approximately when $\omega \approx \frac{4 \pi}{M},|H(\omega)|$ approaches an extremely small number (Oppenheim, 1989). If $M=\frac{4 \pi}{\omega_{d i} T_{s}}$ and $M$ is a positive integer, a smooth shape filter can be generated as $f[k]=\frac{h[k] / e^{\zeta_{i} \omega_{i} k T_{s}}}{\sum_{m=0}^{M} h[m] / e^{\zeta_{i} \omega_{i} m T_{s}}}$. In this case, since $\left|H\left(\omega_{d i} T_{s}\right)\right| \approx 0$, the residual vibration cannot be eliminated in theoretical sense. But an extremely small $\left|H\left(\omega_{d i} T_{s}\right)\right|$ implies the residual vibration is sufficiently suppressed. Again, it is not necessary to derive the explicit form for $f[k]$. Since the sequence $h[k]$ is known, the sequence $f[k]$ can be generated through a simple numerical calculation. Let $f_{1}[k]=f[k]$, then robust shape filters $f_{2}[k], f_{3}[k], \ldots$ can be generated following the filter operation in (1).

\section{SHAPE FILTER GENERATION USING OTHER CONTINUOUS FUNCTIONS}

From Conclusion 2.1, a smooth shape filter can be generated from a finite support smooth function $h(t)$, such that $H\left(\omega_{d i}\right)=0$. Some simple window functions have been used as base functions to generate the shape filters in the previous sections. Numerous smooth functions $h(t)$ may have the property of $H\left(\omega_{d i}\right)=0$ or $H\left(\omega_{d i}\right) \approx 0$. So all of them can be used as base functions to generate the shape filters. Here, some window functions that possess the property are listed: $\cos ^{\alpha}(X)$ window, Riesz window, Riemann window, de la Vallé-Poussin window, Tukey window, Bohman window, Poisson window, Hanning-Poisson window, Cauchy window, Gaussian window, Dolph-Chebeshev window, Kaiser-Bessel window, Barcilon-Temes window, Nuttall window, Modified Bartlett-Hanning window, and Others. These window functions were originally used for harmonic analysis (Harris, 1978), (Nuttall, 1981), (Ha and Pearce, 1989).

\section{SHAPE FILTER GENERATION USING SEVERAL SMOOTH FUNCTIONS}

A section of a base function $h[k]$ can be constructed from other smooth functions through products, sums, convolutions, integral, or other mathematical operations. If the constructed base function has the property of $H\left(\omega_{d i} T_{s}\right)=0$, then it can be used to generate the shape filters through Conclusion 2.1. Here, a simple example is given to show the basic idea.

Here, a base function $h[k]$ is generated from three Hanning window functions $h[k]=h_{s 1}[k]+h_{s 2}[k]+$ $h_{s 3}[k], 0 \leq k \leq 2 M$, where

$h_{s 1}[k]= \begin{cases}\frac{1}{2}-\frac{1}{2} \cos \left(\frac{\pi k}{M}\right), & \text { if } 0 \leq k \leq 2 M, \\ 0, & \text { otherwise, }\end{cases}$
$h_{s 2}[k]= \begin{cases}C\left[\frac{1}{2}-\frac{1}{2} \cos \left(\frac{2 \pi k}{M}\right)\right], & \text { if } 0 \leq k \leq M, \\ 0, & \text { otherwise, }\end{cases}$
$h_{s 3}[k]= \begin{cases}C\left[\frac{1}{2}-\frac{1}{2} \cos \left(\frac{2 \pi k}{M}\right)\right], & \text { if } M \leq k \leq 2 M, \\ 0, & \text { otherwise, }\end{cases}$

and $C$ is a constant number. Fig. 5 shows a typical combination of functions $h_{s 1}[k], h_{s 2}[k], h_{s 3}[k]$, and the resultant base function $h[k]$.

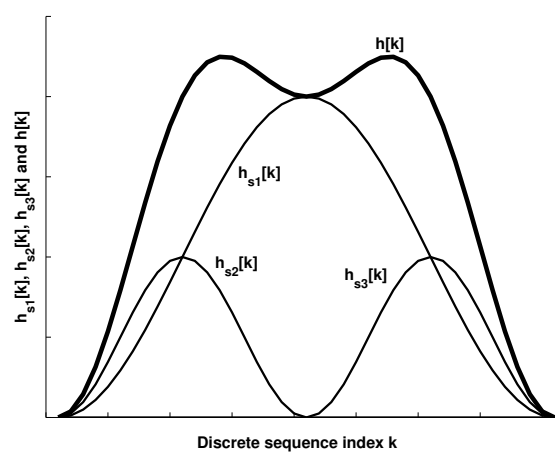

Fig. 5. A typical combination of functions $h_{s 1}[k]$, $h_{s 2}[k], h_{s 3}[k]$, and the resultant base function $h[k]$.

If $H\left(\omega_{d i} T_{s}\right)=H\left(\sqrt{1-\zeta_{i}^{2}} \omega_{i} T_{s}\right)=0$, then $\omega_{d i} T_{s}=$ $\frac{2[\cos (\pi / M)]^{2}(C+1)+2 C \cos (\pi / M)-1}{2 C \cos (\pi / M)+2 C+1}$. So $M$ can be derived to be

$$
M=\frac{\pi}{\arccos \frac{C \cos \left(\omega_{d i} T_{s}\right)-C+\sqrt{B}}{2 C+2}},
$$

where $B=C^{2}\left[\cos \left(\omega_{d i} T_{s}\right)\right]^{2}+2 C^{2} \cos \left(\omega_{d i} T_{s}\right)+$ $C^{2}+2 C+6 C \cos \left(\omega_{d i} T_{s}\right)+2+2 \cos \left(\omega_{d i} T_{s}\right)$. If $M$ is a positive integer, a smooth shape filter can be generated as $f[k]=\frac{h[k] / e^{\zeta_{i} \omega_{i} k T_{s}}}{\sum_{m=0}^{2 M} h[m] / e^{\zeta_{i} \omega_{i} m T_{s}}}, 0 \leq k \leq 2 M$.

Normalized base functions $h[k]$ and shape filters are generated based on the same natural frequency $\omega_{i}=1$ $\mathrm{rad} / \mathrm{sec}$ and the damping ratio $\zeta_{i}=0.2$ with different values of parameter $C$. Fig. 6 shows the normalized base function $h[k]$ and the shape filter $f[k]$ with $C=$ 0.25 . 


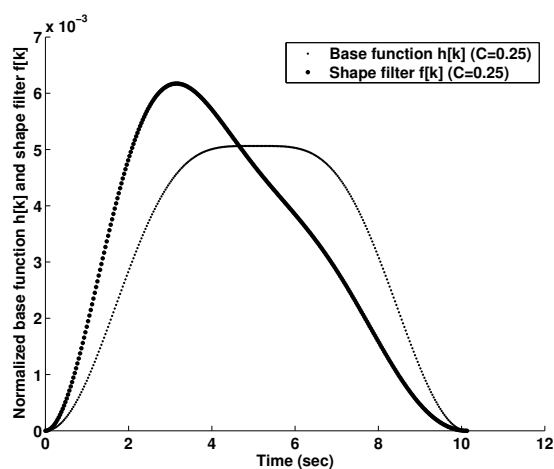

Fig. 6. Normalized base function $h[k]$ and the shape filter $f[k]$ with $C=0.25$.

The value of $M$ in Fig. 6 is made to be a positive integer by choosing appropriate sampling periods. In the derivation, the total impulses of the base function $h[k]$ are assumed to be an odd number $2 M+1$, however, the total impulses of the base function can also be an even number $2 M$.

\section{NON-SYMMETRIC CONTINUOUS FUNCTION BASED SHAPE FILTER GENERATION}

Fig. 7 shows the architecture of the vibration suppression shape filter. From the previous study (Zhou and Misawa, 2005a), the Input Shaping ${ }^{\circledR}$ impulse filters (Singer and Seering, 1990) belong to the impulse function based shape filters. In the previous vibration shape filter generation, all the base functions have a symmetric waveform. In this section, non-symmetric function based shape filter generation method is studied. Two methods to generate a non-symmetric base function are studied.

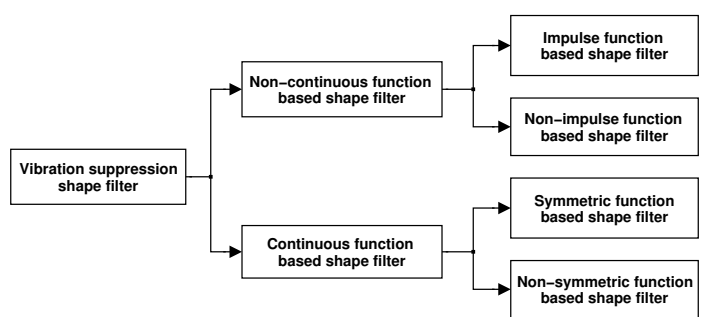

Fig. 7. The architecture of the vibration suppression shape filter.

8.1 Non-Symmetric Base Function Generation from the Derivative of a Base Function

If a discrete-time signal $g[k], 0 \leq k \leq M$, is a base function such that $G\left(\omega_{d i} T_{s}\right)=0$, here $T_{s}$ is the sampling period in sec and $\omega_{d i}$ is the damped natural frequency in $\mathrm{rad} / \mathrm{sec}$, the differencing in time of $g[k]$ is $d g[k]= \begin{cases}g[k]-g[k-1], & \text { if } 0 \leq k \leq M+1 \\ 0, & \text { otherwise }\end{cases}$
A non-symmetric base function $h[k]$ can be generated by the linear combination of $g[k]$ and the differencing signal $d g[k]$ by $h[k]=k_{1} \cdot g[k]+k_{2} \cdot d g[k], 0 \leq$ $k \leq M+1$, where $k_{1}$ and $k_{2}$ are two constants. The discrete-time Fourier transform of $h[k]$ is given by $H(\omega)=k_{1} \cdot G(\omega)+k_{2} \cdot\left(1-e^{-j \omega}\right) G(\omega)$. So the new function $h[k]$ can be used as a base function because the spectrum $H(\omega)$ at $\omega=\omega_{d i} T_{s}$ is exactly zero as given by $H\left(\omega_{d i} T_{s}\right)=k_{1} \cdot G\left(\omega_{d i} T_{s}\right)+k_{2} \cdot(1-$ $\left.e^{-j \omega_{d i} T_{s}}\right) G\left(\omega_{d i} T_{s}\right)=k_{1} \cdot 0+k_{2} \cdot\left(1-e^{-j \omega_{d i} T_{s}}\right) \cdot 0=0$.

A simple example generating a non-symmetric base function is demonstrated here. If $g[k]$ is a Hanning function given in (2), the new generated base function is $h[k]=k_{1} \cdot g[k]+k_{2} \cdot d g[k]$. If $H\left(\omega_{d i} T_{s}\right)=$ $H\left(\sqrt{1-\zeta_{i}^{2}} \omega_{i} T_{s}\right)=0$, then $\omega_{d i} T_{s}=\frac{4 \pi}{M}$ and $M=$ $\frac{4 \pi}{\omega_{d i} T_{s}}$. If $M$ is a positive integer, a shape filter can be generated as $f[k]=\frac{h[k] / e^{\zeta_{i} \omega_{i} k T_{s}}}{\sum_{m=0}^{M} h[m] / e^{\zeta_{i} \omega_{i} m T_{s}}}$.

Fig. 8 shows a construction of a non-symmetric base function $h[k]$ with the undamped natural frequency $\omega_{i}=1 \mathrm{rad} / \mathrm{sec}$ and the damping ratio $\zeta_{i}=0.1$. The sampling period is chosen to be $T_{s}=\frac{\pi}{100 \omega_{d i}}$. The constants $k_{1}$ and $k_{2}$ are chosen to be $k_{1}=1$ and $k_{2}=-25$. Fig. 9 shows the normalized base function $h[k]$ and the shape filter $f[k]$.

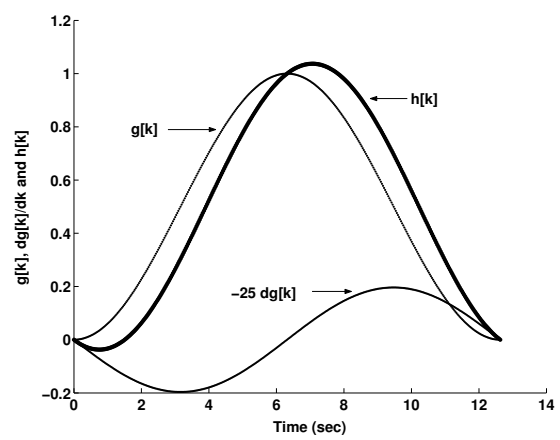

Fig. 8. Construction of a non-symmetric base function $\mathrm{h}[\mathrm{k}]$.

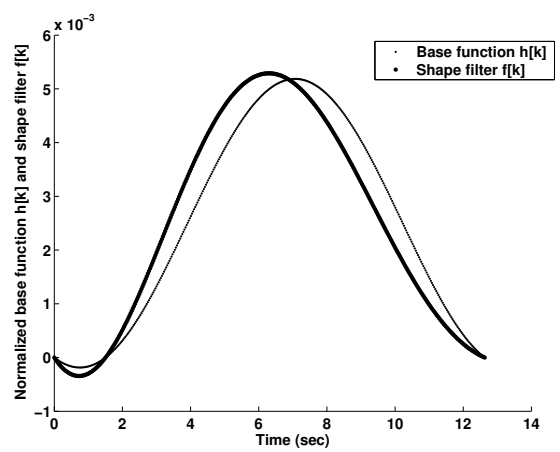

Fig. 9. Normalized base function $h[k]$ and shape filter $f[k]$.

8.2 Non-Symmetric Base Function Generation from the Self Convolution of a Base Function

If a discrete-time signal $g[k], 0 \leq k \leq M$, is a base function such that $G\left(\omega_{d i} T_{s}\right)=0$, here $T_{s}$ is the 
sampling period in sec and $\omega_{d i}$ is the damped natural frequency in $\mathrm{rad} / \mathrm{sec}$, the self convolution of $g[k]$ is $g * g[k]= \begin{cases}\sum_{m=0}^{k} g[k-m] g[m], & \text { if } 0 \leq k \leq 2 M, \\ 0, & \text { otherwise. }\end{cases}$ A non-symmetric base function can be generated by the linear combination of $g[k]$ and the self convolution signal $g * g[k]$ by $h[k]=k_{1} \cdot g\left[k-k_{0}\right]+k_{2} \cdot g * g[k]$, where $k_{0}$ is a non-negative integer. The discrete-time Fourier transform of $h[k]$ is given by $H(\omega)=k_{1}$. $e^{-j \omega k_{0}} G(\omega)+k_{2} \cdot G(\omega)^{2}$. So the new function $h[k]$ can be used as a base function because the spectrum $H(\omega)$ at $\omega=\omega_{d i} T_{s}$ is exactly zero as given by $H\left(\omega_{d i} T_{s}\right)=$ $k_{1} \cdot e^{-j \omega_{d i} T_{s} k_{0}} G\left(\omega_{d i} T_{s}\right)+k_{2} \cdot G\left(\omega_{d i} T_{s}\right)^{2}=k_{1}$. $e^{-j \omega_{d i} T_{s} k_{0}} \cdot 0+k_{2} \cdot 0=0$.

A simple example generating a non-symmetric base function is demonstrated here. If $g[k]$ is a Hanning function given in (2) and $k_{0}=M$, the new generated base function is $h[k]=k_{1} \cdot g[k-M]+k_{2} \cdot g * g[k]$. If $H\left(\omega_{d i} T_{s}\right)=H\left(\sqrt{1-\zeta_{i}^{2}} \omega_{i} T_{s}\right)=0$, then $\omega_{d i} T_{s}=$ $\frac{4 \pi}{M}$ and $M=\frac{4 \pi}{\omega_{d i} T_{s}}$. If $M$ is a positive integer, a shape filter can be generated as $f[k]=\frac{h[k] / e^{\zeta_{i} \omega_{i} k T_{s}}}{\sum_{m=0}^{M} h[m] / e^{\zeta_{i} \omega_{i} m T_{s}}}$.

Fig. 10 shows a construction of a non-symmetric base function $h[k]$ with the undamped natural frequency $\omega_{i}=1 \mathrm{rad} / \mathrm{sec}$ and the damping ratio $\zeta_{i}=0.1$. The sampling period is chosen to be $T_{s}=\frac{\pi}{100 \omega_{d i}}$. The constants $k_{1}$ and $k_{2}$ are chosen to be $k_{1}=1$ and $k_{2}=1 / 125$. Fig. 11 shows the normalized base function $h[k]$ and the shape filter $f[k]$.

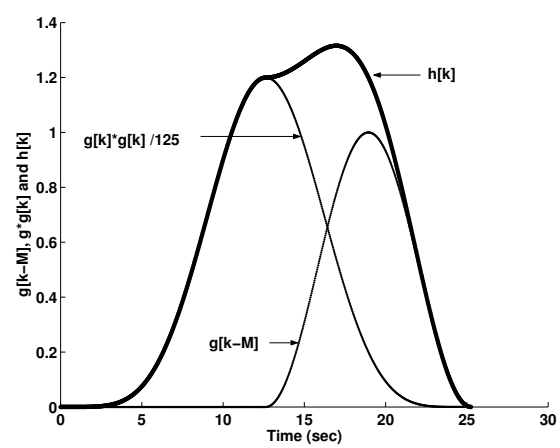

Fig. 10. Construction of a non-symmetric base function $h[k]$.

It must be noted that there are a number of methods to generate a non-symmetric base function. The methods described in this section are only possible methods and not exhaustive ones.

\section{CONCLUSIONS}

A vibration suppression shape filter is generated from a continuous window function. The robustness can be arbitrarily improved and the robustness brings about a smoother profile. The shape filter can also be used as a velocity profile in the case of zero initial and final values. Since the profile is generated from a

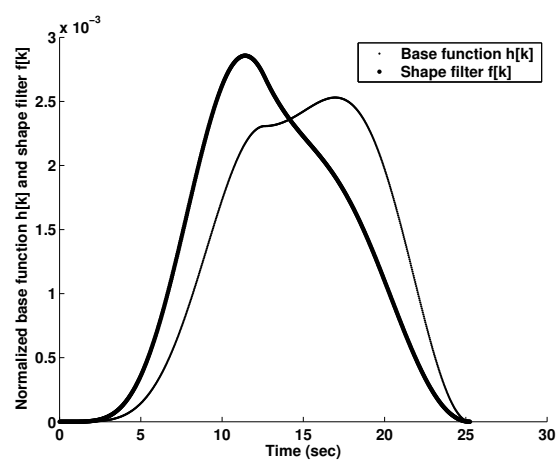

Fig. 11. Normalized base function $h[k]$ and shape filter $f[k]$.

continuous function, a smoothly changing discretetime shape filter can be generated. The methods in this paper were tested on hard disk drive actuator position control at the Oklahoma State University Advanced Controls Laboratory. The experimental results of both Input Shaping ${ }^{\circledR}$ and rectangle based shape filter are reported in (Zhou and Misawa, 2005b). The methods in this paper are patented (pending). Commercial use of these methods requires written permission from the Oklahoma State University.

\section{REFERENCES}

Ha, Y. H. and A. Pearce (1989). A new window and comparison to standard windows. IEEE Transactions on Acoustics, Speech, and Signal Processing 37(2), 298-301.

Harris, F. J. (1978). On the use of windows for harmonic analysis with the discrete Fourier transform. Proceedings of the IEEE 66(1), 51-83.

Nuttall, A. H. (1981). Some windows with very good sidelobe behavior. IEEE Transactions on Acoustics, Speech, and Signal Processing 29(1), 84-91.

Oppenheim, A. V. (1989). Discrete-Time Signal Processing. Prentice Hall, Inc., NJ.

Singer, N. C. and W. P. Seering (1990). Preshaping command inputs to reduce system vibration. ASME, Journal of Dynamic Systems, Measurement, and Control 112, 76-82.

Singhose, W. E. (1997). Command Generation for Flexible Systems. PhD thesis. Massachusetts Institute of Technology.

Zhou, L. (2005). Robust Vibration Suppression Control Profile Generation. PhD thesis. Oklahoma State University.

Zhou, L. and E. A. Misawa (2005a). From Input Shaping $(\mathbb{R}$ and OATF to vibration suppression shape filter. To appear in 2005 American Control Conference.

Zhou, L. and E. A. Misawa (2005b). Low frequency vibration suppression shape filter and high frequency vibration suppression shape filter. To appear in 2005 American Control Conference. 